\title{
ANOTHER PROOF OF A RESULT OF N. J. KALTON, E. SAAB AND P. SAAB ON THE DIEUDONNÉ PROPERTY IN $C(K, E)$
}

\author{
by G. EMMANUELE $\dagger$
}

(Received 27 August, 1987)

1. Let $K$ be a compact Hausdorff topological space and $E$ be a Banach space not containing $l^{1}$. Recently N. J. Kalton, E. Saab and P. Saab ([5]) obtained the results that under the above assumptions the usual space $C(K, E)$ has the Dieudonné property; i.e. each weakly completely continuous operator on $C(K, E)$ is weakly compact. They use topological results concerning multivalued mappings in their proof. In this short note we furnish a new and simpler proof of that result without using topological results but only well known theorems of Bourgain ([2]) and Talagrand ([8]) on weak compactness of sets of Bochner integrable functions; i.e. results in vector measure theory. At the end of the paper we present some applications of the result to Banach spaces of compact operators.

We take this opportunity to thank very much Prof. Paula Saab who suggested the submission of the present proof of her result.

2. In order to give our proof we need a definition and three lemmata. The first one is due to C. Fierro Bello ([4]).

Definition (See [1].) Let $T: C(K, E) \rightarrow F$ be an operator. Then there is a vector measure $m$ from $B o(K)$ into $B(E, F)$ such that

$$
T(f)=\int_{K} f(s) d m
$$

provided $T$ is weakly completely continuous (where $B o(K)$ denotes the $\sigma$-algebra of Borel subsets of $K$ and $B(E, F)$ the Banach space of operators from $E$ into $F$ ). Further it is known that $m$ has a control measure $\lambda$ defined on $B o(K)$. Usually $m$ is named the representing measure of $T$.

Lemma 1 ([4]). Let $\lambda$ be a countably additive, positive and finite Borel measure on $K$. We denote by $\operatorname{cabv}_{\lambda}\left(\mathrm{Bo}(K), E^{*}\right)$ the subspace of all the absolutely $\lambda$-continuous measures in $\operatorname{cabv}\left(B o(K), E^{*}\right)$. If $I: C(K, E) \rightarrow L^{1}(\lambda, E)$ is the canonical embedding and $I^{*}$ the conjugate operator from $\left(L^{1}(\lambda, E)\right)^{*}$ into $\operatorname{cabv}\left(B o(K), E^{*}\right)$ then $\left.I^{*}\left(L^{1}(\lambda, E)\right)^{*}\right)$ is dense in $\operatorname{cabv}_{\lambda}\left(B o(K), E^{*}\right)$.

Moreover we need the other two results contained in Lemma 2 and Lemma 3 in which $T, m$ and $\lambda$ are as in the Definition above.

Lemma 2. Let $\left(g_{n}\right)$ be a sequence in $L^{1}(\lambda, E)$ such that sup sup $\left\|g_{n}(s)\right\|<\infty$ with $g_{n} \stackrel{w}{\longrightarrow} \theta$ in $L^{1}(\lambda, E)$. Then one has $\int_{K} g_{n}(s) d m \stackrel{w}{\longrightarrow} \theta$ in $F$.

† Work performed under the auspices of G.N.A.F.A. of C.N.R. and partially supported by M.P.I. of Italy.

Glasgow Math. J. 31 (1989) 137-140. 
Proof. First of all we observe that $\int_{K} g_{n}(s) d m$ makes sense, for each $n \in \mathbf{N}$, because each $g_{n}$ is $\lambda$-measurable and bounded. Now using Lusin's Theorem ([3]) we can construct a sequence ( $\left.K_{n}\right)$ of compact subsets of $K$ such that (i) $K_{n} \subset K_{n+1}$, (ii) $g_{n}$ restricted to $K_{n}$ (in symbols $\left.g_{n} \mid K_{n}\right)$ is continuous, (iii) the sequence $\left(\lambda\left(K-K_{n}\right)\right.$ ) converges to zero (and then also the sequence $\left(\left\|m\left(K-K_{n}\right)\right\|\right)$ converges to zero). The Borsuk-Dugundji's Theorem ([7]) allows us to define, for each $n \in \mathbf{N}$, an extension operator $S_{n}: C\left(K_{n}, E\right) \rightarrow$ $C(K, E)$ with $\left\|S_{n}\right\|=1$. We have that $S_{n}\left(g_{n} \mid K_{n}\right)(s)=g_{n}(s)$ for all $s \in K_{n}$. Putting $f_{n}=S_{n}\left(g_{n} \mid K_{n}\right) \quad$ we get $\left(f_{n}-g_{n}\right)(s) \stackrel{w}{\longrightarrow} \theta$ a.e. in $K$. From the inequality $\max \left(\sup \sup _{s}\left\|f_{n}(s)\right\|\right.$, sup sup $\left.\left\|g_{n}(s)\right\|\right)<\infty$ we easily get $\left\|f_{n}-g_{n}\right\|_{1} \rightarrow 0$. Thus we have $I\left(f_{n}\right)=f_{n} \stackrel{w}{\longrightarrow} \theta$ in $L^{1}(\lambda, E)$. If $x^{*} \in F^{*}$ we obtain $\left\langle m, x^{*}\right\rangle \in \operatorname{cabv}_{\lambda}\left(B o(K), E^{*}\right)$ and by virtue of Lemma $1 \int_{K} f_{n}(s) d m \stackrel{w}{\longrightarrow} \theta$ in $F$. On the other hand one has

$$
\left\|\int_{K} f_{n}(s)-g_{n}(s) d m\right\| \leqslant \sup _{s \notin K_{n}} \sup _{n}\left\|f_{n}(s)-g_{n}(s)\right\|\left\|m\left(K-K_{n}\right)\right\| \rightarrow 0
$$

from which our result follows.

LeMma 3. Let $\left(h_{n}\right)$ be a sequence in $L^{1}(\lambda, E)$ such that $\sup _{n} \sup _{s}\left\|h_{n}(s)\right\|<\infty$ and $\left(h_{n}(s)\right)$ is a weak Cauchy sequence for all $s \in K$. Then there is $z \in F$ such that $\int_{K} h_{n}(s) d m \stackrel{w}{\longrightarrow} z$.

Proof. Let $\left(\varepsilon_{p}\right)$ be a sequence of positive numbers with $\Sigma \varepsilon_{p}<\infty$. We can construct a sequence $\left(K_{p}\right)$ of compact subsets of $K$ such that (i) $K_{p} \subset K_{p+1}$, (ii) $h_{n} \mid K_{p}=\psi_{n, p}$ is continuous for each $n, p \in \mathbf{N}$, (iii) $\left\|m\left(K-K_{p}\right)\right\|<\varepsilon_{p}$ for each $p \in \mathbf{N}$. Using again the Borsuk-Dugundji's theorem we can define the following functions $\varphi_{n, p}=s_{p}\left(\psi_{n, p}\right)$ for each $n, p \in \mathbf{N}$. Of course $\left(\varphi_{n, p}\right)_{n}$ is a weak Cauchy sequence in $C(K, E)$ for each $p \in \mathbf{N}$. Then for all $p \in \mathbf{N}$ there is a $z_{p} \in F$ for which $T\left(\varphi_{n, p}\right) \stackrel{w}{\longrightarrow} z_{p}$. Now we observe that

$$
\left\|z_{p}-z_{p+1}\right\| \leqslant \lim _{n} \int_{K}\left\|\varphi_{n, p}(s)-\varphi_{n, p+1}(s)\right\| d m \leqslant \text { const. } \varepsilon_{p}
$$

where the constant doesn't depend on $s \in K, n, p \in \mathbf{N}$. Hence the sequence $\left(z_{p}\right)$ must converge to some $z \in F$. It is then easy to see that $\left(T\left(\varphi_{n, n}\right)\right)$ converges weakly to $z$. On the other hand we have

$$
\left\|\int_{K} h_{n}(s)-\varphi_{n, n}(s) d m\right\|=\left\|\int_{K-K_{n}} h_{n}(s)-\varphi_{n, n}(s) d m\right\| \leqslant \text { const. } \varepsilon_{n} \rightarrow 0
$$

Hence we get $\int_{K} h_{n}(s) d m \stackrel{w}{\rightarrow} z$. The proof is complete. 
Now we are able to present our proof of the result in [5].

THEOREM. Let $E$ be a Banach space not containing $l^{1}$. Then $C(K, E)$ has the Dieudonné property.

Proof. Let $\left(f_{n}\right)$ be a bounded sequence in $C(K, E)$. The sequence $\left(I\left(f_{n}\right)\right)=\left(f_{n}\right)$ is uniformly integrable in $L^{1}(\lambda, E)$. A result of Bourgain ([2]) gives that $\left(f_{n}\right)$ is conditionally weakly compact. We may suppose that it is a weak Cauchy sequence; otherwise we pass to a subsequence. Using results by Talagrand ([8]) we have that $f_{n}$ can be written as the sum of two functions $g_{n}$ and $h_{n}$ for each $n \in \mathbf{N}$ and a.e. in $K$. Further from the cited results of [8] it follows that $\left(g_{n}\right)$ converges weakly to $\theta$ and $\left(h_{n}\right)$ may be chosen so that $\left(h_{n}(s)\right)$ is weak Cauchy for each $s \in K$. An inspection of the proof of those results also gives that $\sup _{n} \sup _{s}\left\|g_{n}(s)\right\|<\infty, \sup _{n} \sup _{s}\left\|h_{n}(s)\right\|<\infty$ since this is true for $\left(f_{n}\right)$. Having

$$
\int_{K} f_{n}(s) d m=\int_{K} g_{n}(s) d m+\int_{K} h_{n}(s) d m
$$

we get our thesis by virtue of Lemma 2 and Lemma 3.

The Theorem above has the following consequences relative to Banach spaces of compact operators. If $X$ and $Y$ are two Banach spaces, $K_{w^{*}}\left(X^{*}, Y\right)$ denotes the Banach space of weak* weakly continuous and compact operators from $X^{*}$ into $Y$ equipped with the operator norm ([6]).

Corollary 1. Let $X$ be injective and let $Y$ not contain a copy of $l^{1}$. Then $K_{w^{*}}\left(X^{*}, Y\right)$ has the Dieudonné property.

Proof. $K_{w^{*}}\left(X^{*}, Y\right)$ is isomorphic to $K_{w^{*}}\left(Y^{*}, X\right)$ which is complemented in $K_{w^{*}}\left(Y^{*}, C\left(B_{X^{*}}\right)\right)$ in an obvious way. $\left(B_{X^{*}}\right.$ denotes the unit dual ball of $X$.) This last space is isomorphic to $C\left(B_{X^{*}}, Y\right)$ (see [6]). An application of the Theorem concludes the proof.

In the following corollary $K(X, Y)$ denotes the Banach space of compact operators from $X$ into $Y$ equipped with the operator norm.

Corollary 2. Let $Z$ be an $\mathscr{L}_{\infty}$-space and $Y$ not containing $l^{1}$. Then $K\left(Z^{*}, Y\right)$ has the Dieudonné property.

Proof. $K\left(Z^{*}, Y\right)$ is isomorphic to $K_{w^{*}}\left(Z^{* * *}, Y\right)([6])$. Since it is well known that $Z^{* *}$ is injective an appeal to Corollary 1 concludes the proof.

\section{REFERENCES}

1. J. Batt, G. Berg, Linear bounded transformations on the space of continuous functions, $J$. Functional Analysis 4 (1969), 215-239.

2. J. Bourgain, An averaging result for $l^{1}$ sequences and applications to weakly conditionally compact subsets in $L_{X}^{\prime}$, Israel J. Math. 32 (1979), 289-298.

3. N. Dinculeanu, Vector measures (Pergamon Press, 1967). 
4. C. Fierro Bello, On weakly compact and unconditionally converging operators in spaces of vector valued continuous functions, preprint.

5. N. J. Kalton, E. Saab, P. Saab, On the Dieudonné property for $C(K, E)$, Proc. Amer. Math. Soc. 96 (1986), 50-52.

6. W. Ruess, Duality and geometry of spaces of compact operators, Math. Studies 90 (North Holland, 1984).

7. Z. Semadeni, Banach spaces of continuous functions (P. W. N. Warszawa, 1971).

8. M. Talagrand, Weak Cauchy sequences in $L^{\prime}(E)$, Amer. J. Math. 106 (1984), 703-724.

Department of Mathematics

UNIVERSITY OF CATANIA

Viale A. DoRia 6

Catania 95125

ITALY 